\title{
A reduced basis method applied to the Restricted Hartree-Fock equations
}

\author{
Yvon Maday ${ }^{1,2}$, Ulrich Razafison ${ }^{1}$ \\ 1 - Université Pierre et Marie Curie-Paris6, UMR 7598, Laboratoire J.-L. Lions, Paris, F-75005 France \\ 2 - Division of Applied Mathematics, Brown University 182 George Street, Providence, RI 02912, USA
}

\begin{abstract}
In this Note, we describe a reduced basis approximation method for the computation of some electronic structure in quantum chemistry, based on the Restricted Hartree-Fock equations. Numerical results are presented to show that this approach allows for reducing the complexity and potentially the computational costs.
\end{abstract}

\section{Résumé}

Dans cette Note, nous décrivons une méthode d'approximation par bases réduites pour les calculs de structures électroniques en chimie quantique basées sur le modèle Restricted Hartree-Fock. Nous présentons des résultats numériques montrant que la méthode permet des réductions de complexité et potentiellement de coûts de calculs.

\section{Version française abrégée}

Nous considérons le calcul de l'état fondamental électronique d'une molécule composée de $M$ noyaux de charges électriques $z_{1}, \ldots, z_{M}$ situés en $\overline{\boldsymbol{x}}_{1}, \ldots, \overline{\boldsymbol{x}}_{M}$ dans $\mathbb{R}^{3}$ et de $2 n_{e}$ électrons. Sous l'approximation de Born-Oppenheimer et du modèle Restricted Hartree-Fock, le problème s'écrit comme le problème de minimisation (1). Les équations d'Euler-Lagrange associées à ce problème consistent à trouver $(\boldsymbol{\Phi}, \boldsymbol{\lambda}) \in$ $\left(H^{1}\left(\mathbb{R}^{3}\right)\right)^{n_{e}} \times \mathbb{R}^{n_{e}\left(n_{e}+1\right) / 2}$ satisfaisant (2). Dans cette Note, nous proposons une méthode de bases réduites pour l'approximation de ces équations. En posant $\mu \equiv\left(\overline{\boldsymbol{x}}_{1}, \ldots, \overline{\boldsymbol{x}}_{M}\right)$, où le paramètre $\mu \in \mathcal{D} \subset \mathbb{R}^{3 M}$, la méthode consiste tout d'abord à séléctionner un échantillon de paramètres $\mu_{1}, \ldots, \mu_{N}$, ensuite à calculer les solutions correspondantes $\boldsymbol{\Phi}\left(\mu_{k}\right) \equiv\left(\varphi_{1}\left(\mu_{k}\right), \ldots, \varphi_{n_{e}}\left(\mu_{k}\right)\right)$ et enfin à chercher une approximation d'une solution $\boldsymbol{\Phi}$ correspondant à un nouveau paramètre $\mu$ comme une combinaison linéaire des $\boldsymbol{\Phi}\left(\mu_{k}\right), k=$ $1, \ldots, N$. Comme (2) revient à résoudre un problème non linéaire aux valeurs propres, il y a ici deux approches possibles : l'approche mode par mode, c'est-à-dire, $\varphi_{i}(\mu) \simeq \sum_{k=1}^{N} \beta_{i}^{k}(\mu) \varphi_{i}\left(\mu_{k}\right)$ et l'approche vectorielle $\boldsymbol{\Phi}(\mu) \simeq \sum_{k=1}^{N} \beta^{k}(\mu) \boldsymbol{\Phi}\left(\mu_{k}\right)$, où $\beta^{k}(\mu)$ ne dépend pas de $i$. Il est important de remarquer que

Email addresses: maday@ann.jussieu.fr (Yvon Maday ${ }^{1,2}$ ), razafison@ann.jussieu.fr (Ulrich Razafison ${ }^{1}$ ). 
nous cherchons les $n_{e}$ fonctions propres $\varphi_{i}\left(\mu_{k}\right)$ associées aux $n_{e}$ premières valeurs propres rangées par ordre croissant. À cause de croisements possibles de valeurs propres, pour deux valeurs proches $\mu$ et $\mu^{*}$ du paramètre, la fonction $\varphi_{i}\left(\mu^{*}\right)$ peut être plus proche de $\varphi_{i+1}(\mu)$ que de $\varphi_{i}(\mu)$; de même $+\varphi(\mu)$ et $-\varphi(\mu)$ sont des fonctions propres possibles. On propose donc tout d'abord une procédure pour classer et orienter les fonctions propres par similarité. L'approximation par bases réduites s'écrit ensuite sous la forme (3) où, comparant avec (2), on peut remarquer que nous n'avons imposé que les contraintes de normalité et, si $\varepsilon=1$ les contraintes d'orthogonalité adjascentes. Les tableaux 1 et 2 montrent les résultats de meilleure approximation obtenus sur la molécule d'eau par les deux approches de la méthode, la solution approchée $\boldsymbol{\Phi}_{N}(\mu)$ est obtenu par la projection de $\boldsymbol{\Phi}(\mu)$ sur les espaces bases réduites. Ces résultats préliminaires montrent que l'approche vectorielle induit un plus faible nombre de degrés de liberté et on choisit cette approche pour obtenir cette fois $\boldsymbol{\Phi}_{N}(\mu)$ par la résolution (3). Le Tableau 3 montre une convergence d'ordre 9 de l'approximation. Dans le tableau 4 , on considère la réaction entre l'ion $\mathrm{F}^{-}$et la molécule de méthane $\mathrm{CH}_{4}: \mathrm{F}^{-}+\mathrm{CH}_{4} \rightarrow \mathrm{CH}_{3} \mathrm{~F}^{-}+\mathrm{H}$. Pour ce problème, nous avons $\varepsilon=1$ dans (3). Le tableau 4 montre que la convergence est aussi ici de l'ordre de 9.

\section{Introduction}

Many problems in computational quantum chemistry (such as geometry optimization or ab initio molecular dynamics) require the repetitive evaluation of "outputs", such as the energies of the system. These outputs depend on input parameters denoted as $\mu \in \mathcal{D} \subset \mathbb{R}^{p}$ that reflect the configuration of the system (e.g. the positions of the nuclei). In ab initio quantum models (such as Hartree-Fock, Multiconfigurations, Density Functional Theory), the outputs are typically functions of a field variable $u(\mu)$, a wave function or a set of molecular orbitals, which satisfy one or a set of partial differential equations in which the parameters $\mu$ enter. The approximation of $u(\mu)$ with standard numerical approaches (plane waves, LCAO or even finite element methods) is most of the time very expensive and has to be repeated for any input parameter. The approach presented in this Note, is based on a reduced-basis method (see for instance [7]). The method exploits the fact that, although each field $u(\mu)$ belongs individually to an infinite-dimensional space (often $Y \equiv H^{1}\left(\mathbb{R}^{3}\right)$ ), the set $\{u(\mu), \mu \in \mathcal{D}\}$ is close to a low-dimensional manifold. The main idea is then to consider a new space of approximation spanned by a small number of well chosen solutions $u\left(\mu_{k}\right)$. This approach has been successfully applied to parameterized partial differential equations and eigenvalues problems in many engineering sciences [6] [11] [12]. We extend here the reduced-basis approach to the computation of electronic structures in quantum chemistry, based on the Restricted Hartree-Fock equations. The preliminary results we report illustrate an important reduction in the number of degrees of freedom for the approximation strategy yielding a potential large speed up in the computations.

The application of the reduced basis method to the context of computational chemistry was first proposed in [3] (see also [1]). We refer in particular to [5], [8] for an application to DFT for cristals.

\section{Problem statement}

We consider the computation of the ground state energy of a system of $M$ nuclei with charge $z_{1}, \ldots, z_{M}$ located in $\overline{\boldsymbol{x}}_{1}, \ldots, \overline{\boldsymbol{x}}_{M}$ in $\mathbb{R}^{3}$ and $2 n_{e}$ electrons. Within the Born-Oppenheimer approximation and the Restricted Hartree-Fock (RHF) model, this problem writes as the following minimization problem:

$$
\inf \left\{E^{R H F}\left(\boldsymbol{\Phi} \equiv\left(\varphi_{1}, \ldots, \varphi_{n_{e}}\right)\right), \varphi_{i} \in H^{1}\left(\mathbb{R}^{3}\right), \int_{\mathbb{R}^{3}} \varphi_{i} \varphi_{j}=\delta_{i j}, 1 \leq i, j \leq n_{e}\right\},
$$


with $E^{R H F}(\boldsymbol{\Phi})=\sum_{i=1}^{n_{e}} \int_{\mathbb{R}^{3}}\left|\nabla \varphi_{i}\right|^{2}+\int_{\mathbb{R}^{3}} \rho_{\Phi} V+\frac{1}{2} \int_{\mathbb{R}^{3}} \int_{\mathbb{R}^{3}} \frac{\rho_{\Phi}(\boldsymbol{x}) \rho_{\Phi}(\boldsymbol{y})}{|\boldsymbol{x}-\boldsymbol{y}|} d \boldsymbol{x} d \boldsymbol{y}-\frac{1}{4} \int_{\mathbb{R}^{3}} \int_{\mathbb{R}^{3}} \frac{\left|\tau_{\Phi}(\boldsymbol{x}, \boldsymbol{y})\right|^{2}}{|\boldsymbol{x}-\boldsymbol{y}|} d \boldsymbol{x} d \boldsymbol{y}$, and where the density matrix $\tau_{\Phi}(\boldsymbol{x}, \boldsymbol{y})$, the electronic density $\rho_{\Phi}(\boldsymbol{x})$ and the potential $V$ are respectively given by $\tau_{\Phi}(\boldsymbol{x}, \boldsymbol{y})=2 \sum_{i=1}^{n_{e}} \varphi_{i}(\boldsymbol{x}) \varphi_{i}(\boldsymbol{y}), \rho_{\Phi}(\boldsymbol{x})=\tau_{\Phi}(\boldsymbol{x}, \boldsymbol{x}), \quad V(\boldsymbol{x})=-\sum_{k=1}^{M} \frac{z_{k}}{\left|\boldsymbol{x}-\overline{\boldsymbol{x}}_{k}\right|}$. Defining $Y \equiv\left(H^{1}\left(\mathbb{R}^{3}\right)\right)^{n_{e}}$, the Euler-Lagrange equations associated with (1) consist in finding $(\boldsymbol{\Phi}, \boldsymbol{\lambda}) \in Y \times \mathbb{R}^{\frac{n_{e}\left(n_{e}+1\right)}{2}}$ such that,

$$
\begin{aligned}
& \forall(\boldsymbol{\psi}, \boldsymbol{\omega}) \in Y \times \mathbb{R}^{\frac{n_{e}\left(n_{e}+1\right)}{2}}, \sum_{i=1}^{n_{e}}\left[\frac{1}{2} \int_{\mathbb{R}^{3}} \nabla \varphi_{i} \nabla \psi_{i}+\int_{\mathbb{R}^{3}} V \varphi_{i} \psi_{i}+2 \sum_{j=1}^{n_{e}} \int_{\mathbb{R}^{3}} \int_{\mathbb{R}^{3}} \frac{\left|\varphi_{j}(\boldsymbol{y})\right|^{2} \varphi_{i}(\boldsymbol{x}) \psi_{i}(\boldsymbol{x})}{|\boldsymbol{x}-\boldsymbol{y}|} d \boldsymbol{x} d \boldsymbol{y}\right. \\
& \left.-\sum_{j=1}^{n_{e}} \int_{\mathbb{R}^{3}} \int_{\mathbb{R}^{3}} \frac{\varphi_{i}(\boldsymbol{y}) \varphi_{j}(\boldsymbol{y}) \varphi_{j}(\boldsymbol{x}) \psi_{i}(\boldsymbol{x})}{|\boldsymbol{x}-\boldsymbol{y}|} d \boldsymbol{x} d \boldsymbol{y}-\sum_{j=1}^{n_{e}} \lambda_{i j} \int_{\mathbb{R}^{3}} \varphi_{i} \psi_{j}\right]+\sum_{i=1}^{n_{e}} \sum_{j=i}^{n_{e}} \omega_{i j}\left(\int_{\mathbb{R}^{3}} \varphi_{i} \varphi_{j}-\delta_{i j}\right)=0 .
\end{aligned}
$$

\section{Reduced basis approximation}

Let us set $\mu \equiv\left(\overline{\boldsymbol{x}}_{1}, \ldots, \overline{\boldsymbol{x}}_{M}\right)$ and assume that we are given a subset $\mathcal{D} \subset \mathbb{R}^{3 M}$ in which $\mu$ varies. The reduced basis approximation consists first in providing appropriate countable (and ordered) set $\Sigma_{\mu} \subset \mathcal{D}$, then in considering the associated solutions $\boldsymbol{\Phi}\left(\mu_{k}\right)=\left(\varphi_{1}\left(\mu_{k}\right), \ldots, \varphi_{n_{e}}\left(\mu_{k}\right)\right), \mu_{k} \in \Sigma_{\mu}$ finally in looking for an approximation of the solution $\boldsymbol{\Phi}$ corresponding to a new parameter value $\mu \in \mathcal{D}$ as a linear combination of the $\boldsymbol{\Phi}\left(\mu_{k}\right), k=1, \ldots, N$. The bottom line for the reduced basis method is to exploit the (most often) rapidly decreasing $N$-width (see for instance [9]) of the set of all solutions $\{\boldsymbol{\Phi}(\mu), \mu \in \mathcal{D}\}$.

\subsection{Offline construction of the reduced basis spaces}

In practice, the solutions $\boldsymbol{\Phi}\left(\mu_{k}\right)$ are obtained by a numerical approximation of Equation (2). In our case, this resolution is based on the LCAO approach which consists in approximating each function $\varphi_{i}(\mu)$, for $1 \leq i \leq n_{e}$ by linear combination of a large number, say $\mathcal{N}$, of atomic orbitals.

As (2) is a (nonlinear) eigenvalue problem, we can propose two approaches for the construction of the reduced space: the mode-by-mode approximation where $\varphi_{i}(\mu) \simeq \sum_{k=1}^{N} \beta_{i}^{k}(\mu) \varphi_{i}\left(\mu_{k}\right)$ and the vectorial approximation where $\varphi_{i}(\mu) \simeq \sum_{k=1}^{N} \beta^{k}(\mu) \varphi_{i}\left(\mu_{k}\right)$ (note that $\beta^{k}(\mu)$ then do not depend on $i$ ). An important remark is the following: we know (for a theoretical result see [4]) that we search for the $n_{e}$ eigenfunctions $\varphi_{i}\left(\mu_{k}\right)$ associated with the first $n_{e}$ eigenvalues ranked in increasing order. Due to possible mode crossings, it may be the case that for two close values of the parameter, say $\mu$ and $\mu^{*}, \varphi_{i}\left(\mu^{*}\right)$ is closer to $\varphi_{i+1}(\mu)$ than to $\varphi_{i}(\mu)$. For both variants of the method it is thus important to sort the eigenfunctions by similarity. For the vectorized approximation, it is also important to respect the good orientation since $+\varphi_{i}(\mu)$ and $-\varphi_{i}(\mu)$ are possible eigenvectors. The alignment procedure we have implemented follows the approach used in [8]. We choose a reference solution $\mathbf{\Phi}\left(\mu_{r e f}\right)=\left(\varphi_{1}\left(\mu_{r e f}\right), \ldots, \varphi_{n_{e}}\left(\mu_{r e f}\right)\right)$ where the parameter $\mu_{\text {ref }} \in \Sigma_{\mu}$. For any other solution $\boldsymbol{\Phi}\left(\mu_{i}\right)$, where $\mu_{i} \in \Sigma_{\mu}$ and $\mu_{i} \neq \mu_{\text {ref }}$, we compute $e_{j}=\left(\varphi_{1}\left(\mu_{r e f}\right), \varphi_{j}\left(\mu_{i}\right)\right)_{L^{2}\left(\mathbb{R}^{3}\right)}$, $j=1, \ldots, n_{e}$. We then determine $j_{\max }=\arg \max _{1 \leq j \leq n_{e}}\left|e_{j}\right|$. If $\left(\varphi_{1}\left(\mu_{r e f}\right), \varphi_{j_{\max }}\left(\mu_{i}\right)\right)>0$, then $\left(\boldsymbol{\Phi}\left(\mu_{i}\right)\right)_{1}=$ $\varphi_{j_{\max }}\left(\mu_{i}\right)$, otherwise $\left(\boldsymbol{\Phi}\left(\mu_{i}\right)\right)_{1}=-\varphi_{j_{\max }}\left(\mu_{i}\right)$. This is then repeated for all $\left(\boldsymbol{\Phi}\left(\mu_{i}\right)\right)_{j}$, with $j=2, \ldots, n_{e}$.

We now explain (on the vectorial case) the issue related to the choice of the parameter set $S_{N}$ and the construction of the corresponding reduced-basis space $W_{N}$. It is based on an algorithm outlined in [10]. Given a reduced basis space $W_{N}=\operatorname{span}\left\{\boldsymbol{\Phi}\left(\mu_{k}\right), 1 \leq k \leq N\right\}=\operatorname{span}\left\{\boldsymbol{\xi}_{k}, 1 \leq k \leq N\right\}$, we consider the next parameter $\mu_{N+1}$ defined by $\mu_{N+1}=\arg \max _{\mu \in \Sigma_{\mu}}\left\|\boldsymbol{\Phi}(\mu)-\sum_{k=1}^{N}\left(\boldsymbol{\Phi}(\mu), \boldsymbol{\xi}_{k}\right)_{Y} \boldsymbol{\xi}_{k}\right\|_{Y}$. We next compute $\boldsymbol{u}=\boldsymbol{\Phi}\left(\mu_{N+1}\right)-\sum_{k=1}^{N}\left(\boldsymbol{\Phi}\left(\mu_{N+1}\right), \boldsymbol{\xi}_{k}\right)_{Y} \boldsymbol{\xi}_{k}$ and the new pseudo-orthogonalized basis is then given by $\boldsymbol{\xi}_{N+1}=\boldsymbol{u} /\|\boldsymbol{u}\|_{Y}$, the new space is then $W_{N+1}=\operatorname{span}\left\{\boldsymbol{\xi}_{1}, \ldots, \boldsymbol{\xi}_{N+1}\right\}$. 
The computation strategy is based on a offline-online decomposition. The offline stage that we just sketched, is performed once and is mainly devoted to the construction of the reduced basis: we first compute the solutions $\boldsymbol{\Phi}\left(\mu_{k}\right), \mu_{k} \in \sum_{\mu}$. Next, these solutions are preprocessed by the procedure described above and many scalar products are computed at this stage. We then construct the reduced basis $W_{N}$.

\subsection{Preliminary verifications}

We illustrate here the ability of the reduced basis space to approximate well $\boldsymbol{\Phi}(\mu)$ for different values of $\mu$. We consider the water molecule $\mathrm{H}_{2} \mathrm{O}\left(n_{e}=5\right)$ and use the code ASPIC (developed in a collaboration between J.L. Lions Lab. and Cermics at École Nationale des Ponts et Chaussées, France) to approximate a large set of solutions based on a LCAO basis $(6-31 \mathrm{G})$ with $\mathcal{N}=13$ atomic orbitals. The parameter is $\mu=(\theta, d)$ where $\theta$ is the $\mathrm{H}-\mathrm{O}-\mathrm{H}$ angle and $d$ is one of the $\mathrm{O}-\mathrm{H}$ distance, the other one being fixed equal to $0.9 \AA$. A parameter set $\Sigma_{\mu}$ of size 100 is designed with $80^{\circ} \leq \theta \leq 130^{\circ}$ and $0.5 \AA \leq d<2 \AA$. We then define recursively the reduced basis as explained above after properly sorting the eigenvectors and look for the best approximation $\boldsymbol{\Phi}_{N}(\mu)$ through a Hilbert projection over $W_{N}$ for each of the solutions $\boldsymbol{\Phi}(\mu)$ previously computed. We then define $e^{E}=\max _{\mu \in \Sigma_{\mu}}\left|E^{R H F}(\boldsymbol{\Phi}(\mu))-E^{R H F}\left(\boldsymbol{\Phi}_{N}(\mu)\right)\right| /\left|E^{R H F}(\boldsymbol{\Phi}(\mu))\right|$ and $e^{\boldsymbol{\Phi}}=\max _{\mu \in \Sigma_{\mu}}\left\|\boldsymbol{\Phi}(\mu)-\boldsymbol{\Phi}_{N}(\mu)\right\|_{Y} /\|\boldsymbol{\Phi}(\mu)\|_{Y}$. In Table 1, we have used the mode-by-mode approach to construct our best approximations whereas in Table 2 we have used the vectorized approach. Comparing Table 1 and 2, we first observe that the vectorized reduced-basis approach requires only 8 basis functions to provide $e^{\boldsymbol{\Phi}} \approx 10^{-5}$, whereas the mode by mode approach requires $30\left(=6 \times n_{e}\right)$ basis functions for the same accuracy. We first deduce that the vectorized approach provides smaller numbers of degrees of freedom for a given accuracy. Then we notice that doubling the number of degrees of freedom allows to diminish the errors $e^{\boldsymbol{\Phi}}$ by a factor $5.10^{2}$ meaning that the order of the approximation is about 9 .

Table 1

Variation of the reduced-basis errors of the mode by mode approach with $\boldsymbol{\Phi}_{N}$ obtained by projection

\begin{tabular}{|c|c|c|c|c|c|}
\hline$N$ & 2 & 3 & 4 & 5 & 6 \\
\hline$e^{\Phi}$ & $2.2831 E-02$ & $7.8354 E-03$ & $3.3562 E-03$ & $2.1117 E-04$ & $4.878 E-05$ \\
\hline$e^{E}$ & $3.6766 E-03$ & $4.5661 E-05$ & $1.6178 E-04$ & $2.0178 E-05$ & $5.8735 E-07$ \\
\hline$e^{\text {ortho }}$ & $8.9471 E-02$ & $1.9975 E-02$ & $8.8881 E-03$ & $2.9562 E-04$ & $9.2915 E-05$ \\
\hline
\end{tabular}

Table 2

Variation of the reduced-basis errors of the vectorized approach with $\boldsymbol{\Phi}_{N}$ obtained by projection

\begin{tabular}{|c|c|c|c|c|c|c|c|c|}
\hline$N$ & 5 & 6 & 7 & 8 & 10 & 12 & 14 & 16 \\
\hline$e^{\Phi}$ & $2.7533 E-03$ & $7.1008 E-04$ & $3.7623 E-04$ & $4.9208 E-05$ & $8.4117 E-06$ & $1.1479 E-06$ & $3.8195 E-07$ & $1.0037 E-07$ \\
\hline$e^{E}$ & $1.7952 E-04$ & $1.2726 E-04$ & $2.5236 E-06$ & $7.3068 E-07$ & $3.3172 E-07$ & $8.1656 E-08$ & $3.4266 E-08$ & $8.1925 E-08$ \\
\hline$e^{\text {ortho }}$ & $2.1606 E-03$ & $1.1656 E-03$ & $5.858 E-04$ & $7.3193 E-05$ & $5.5197 E-06$ & $2.226 E-06$ & $9.4677 E-07$ & $1.4958 E-07$ \\
\hline
\end{tabular}

Finally, even though the Hilbert projection was done without any orthogonality constraint, we verify that it is rather well approximated, indeed the quantity $e^{\text {ortho }}=\max _{\mu \in \Sigma_{\mu}} \max _{1 \leq i<j \leq n_{e}}\left(\varphi_{i}(\mu), \varphi_{j}(\mu)\right)_{L^{2}\left(\mathbb{R}^{3}\right)}$ decreases rapidly. This leads us to consider that the orthogonality conditions may not be so important in the approximation, they come for free...

\subsection{Discrete problem}

Our discrete problem originates from the variational formulation (2) where we remark that the last contribution states the orthonormality of the wavefunctions. Let $\varepsilon$ be an integer that we can take equal 
to 0 or 1 , the reduced-basis approximation is:

find $\left(\boldsymbol{\Phi}_{N}(\mu), \boldsymbol{\lambda}_{N}(\mu)\right) \in W_{N} \times \mathbb{R}^{n_{e}+\varepsilon\left(n_{e}-1\right)}$ such that for any $(\boldsymbol{\psi}, \boldsymbol{\omega}) \in W_{N} \times \mathbb{R}^{n_{e}+\varepsilon\left(n_{e}-1\right)}$,

$$
\begin{aligned}
& \sum_{i=1}^{n_{e}}\left[\frac{1}{2} \int_{\mathbb{R}^{3}} \nabla \varphi_{N, i} \nabla \psi_{i}+\int_{\mathbb{R}^{3}} V \varphi_{N, i} \psi_{i}+2 \sum_{j=1}^{n_{e}} \int_{\mathbb{R}^{3}} \int_{\mathbb{R}^{3}} \frac{\left|\varphi_{N, j}(\boldsymbol{y})\right|^{2} \varphi_{N, i}(\boldsymbol{x}) \psi_{i}(\boldsymbol{x})}{|\boldsymbol{x}-\boldsymbol{y}|} d \boldsymbol{x} d \boldsymbol{y}\right. \\
& \left.-\sum_{j=1}^{n_{e}} \int_{\mathbb{R}^{3}} \int_{\mathbb{R}^{3}} \frac{\varphi_{N, i}(\boldsymbol{y}) \varphi_{N, j}(\boldsymbol{y}) \varphi_{N, j}(\boldsymbol{x}) \psi_{i}(\boldsymbol{x})}{|\boldsymbol{x}-\boldsymbol{y}|} d \boldsymbol{x} d \boldsymbol{y}-\lambda_{N, i i} \int_{\mathbb{R}^{3}} \varphi_{N, i} \psi_{i}\right]+\sum_{i=1}^{n_{e}} \omega_{i i}\left(\int_{\mathbb{R}^{3}}\left|\varphi_{N, i}\right|^{2}-1\right) \\
& +\varepsilon\left[\sum_{i=1}^{n_{e}-1}-\lambda_{N, i i+1} \int_{\mathbb{R}^{3}} \varphi_{N, i} \psi_{i+1}+\omega_{i i+1} \int_{\mathbb{R}^{3}} \varphi_{N i} \varphi_{N i+1}\right]=0,
\end{aligned}
$$

where $\boldsymbol{\Phi}_{N}(\mu) \equiv\left(\varphi_{N, i}(\mu), 1 \leq i \leq n_{e}\right)$ and $\boldsymbol{\lambda}_{N}(\mu) \equiv\left(\lambda_{N, 11}(\mu), \ldots, \lambda_{N, n_{e} n_{e}}, \varepsilon \lambda_{N, 12}, \ldots, \varepsilon \lambda_{N, n_{e}-1 n_{e}}\right)$. When compared to (2), we note that we have chosen to impose only the normalization constraints $\int_{\mathbb{R}^{3}}\left|\varphi_{N, i}\right|^{2}=1$, $1 \leq i \leq n_{e}$ together eventually the adjacent orthogonality constraints $\int_{\mathbb{R}^{3}} \varphi_{N, i} \varphi_{N, i+1}=0$, by choosing $\varepsilon=1$ in (3). The actual dimension of the algebraic system is then $N+n_{e}+\varepsilon\left(n_{e}-1\right)$.

We use a Newton's method to solve the algebraic system coming from (3).

\section{Numerical results}

\subsection{The water molecule}

We report in Table 3 the results for the reduced basis method used to solve equation (3) with $\varepsilon=0$. When comparing with Table 2, we see that the numerical solution behaves similarly as the best fit.

Table 3

Variation of the reduced-basis errors of the vectorized approach with $\boldsymbol{\Phi}_{N}$ obtained by solving (3)

\begin{tabular}{|c|c|c|c|c|c|c|c|c|}
\hline$N$ & 5 & 6 & 7 & 8 & 10 & 12 & 14 & 16 \\
\hline$e^{\boldsymbol{\Phi}}$ & $7.2655 E-02$ & $3.7257 E-03$ & $1.9989 E-03$ & $4.3774 E-04$ & $1.192 E-04$ & $3.001 E-05$ & $8.819 E-07$ & $1.5368 E-07$ \\
\hline$e^{E}$ & $7.9006 E-04$ & $1.5607 E-06$ & $4.2416 E-08$ & $8.5609 E-09$ & $7.9263 E-11$ & $2.0499 E-12$ & $5.2959 E-15$ & $1.4914 E-15$ \\
\hline$e^{\text {ortho }}$ & $6.1852 E-02$ & $6.4796 E-03$ & $2.8667 E-03$ & $9.8977 E-04$ & $1.0759 E-04$ & $1.3558 E-05$ & $8.439 E-07$ & $1.4482 E-07$ \\
\hline
\end{tabular}

\subsection{A more complex case}

In the second test, we consider the reaction between the ion $\mathrm{F}^{-}$and the methane molecule $\mathrm{CH}_{4}$ : $\mathrm{F}^{-}+\mathrm{CH}_{4} \rightarrow \mathrm{CH}_{3} \mathrm{~F}^{-}+\mathrm{H}$. Here the number of pair of electrons is $n_{e}=9$. The LCAO basis is again 6 -31G with now $\mathcal{N}=26$ atomic orbitals. We consider the parameter $\mu=\left(\theta, d_{F}, d_{H}, d\right)$ where, $\theta$ is the $\mathrm{H}-\mathrm{C}-\mathrm{H}$ angle, $d_{F}$ is the $\mathrm{F}-\mathrm{C}$ distance, $d_{H}$ is one $\mathrm{C}-\mathrm{H}$ distance and $d$ is the other $\mathrm{C}-\mathrm{H}$ distances that we assume identical. The parameter set $\Sigma_{\mu}$ is of size 100 with $87^{\circ} \leq \theta \leq 109.5^{\circ}, 1.15 \AA \leq d_{F} \leq 1.5 \AA$, $1.091 \AA \leq d_{H} \leq 1.5 \AA$ and $1.082 \AA \leq d \leq 1.091 \AA$. Here we include some orthogonality constraints by choosing $\varepsilon=1$. We again observe a convergence order for the errors about equal to 9 . 
Table 4

Variation of the reduced-basis error with $\boldsymbol{\Phi}_{N}$ obtained by resolving (3)

\begin{tabular}{|c|c|c|c|c|c|c|c|c|}
\hline$N$ & 18 & 19 & 20 & 21 & 36 & 38 & 40 & 42 \\
\hline$e^{\boldsymbol{\Phi}}$ & $1.2407 E-01$ & $6.5046 E-03$ & $1.0888 E-03$ & $5.4543 E-03$ & $1.6311 E-04$ & $2.0232 E-05$ & $6.6913 E-05$ & $1.7891 E-05$ \\
\hline$e^{E}$ & $8.3389 E-05$ & $5.3251 E-06$ & $1.1965 E-06$ & $1.4195 E-07$ & $9.8293 E-11$ & $1.6531 E-12$ & $3.9346 E-12$ & $3.1278 E-12$ \\
\hline$e^{\text {ortho }}$ & $1.2407 E-01$ & $7.5226 E-03$ & $1.901 E-03$ & $8.0144 E-03$ & $1.167 E-04$ & $1.1855 E-05$ & $7.393 E-05$ & $1.6658 E-05$ \\
\hline
\end{tabular}

\section{Conclusion and next steps}

The numerical results presented in this Note are encouraging and indicate that the reduced basis method may be successfully applied for solving the Restricted Hartree-Fock equations. The current implementation does not exploit fully all the features of the reduced-basis methodology to make the implementation fully independent of $\mathcal{N}$. Provided that the offline step include the computation of some scalar products and we use the magic point approach to evaluate the nonlinear contributions (see [2]) the operation count of the online stage is $O\left(N^{3}+n_{e} N^{2}\right)$. In addition, we need to develop a posteriori error estimators in order to evaluate the accuracy of our approximation.

Acknowledgements. We would like to thank É. Cancès and C. Le Bris from CERMICS-ENPC, together with A. P. Patera and G. Pau from MIT, for many helpful comments and careful critiques of the paper.

\section{References}

[1] M. Barrault, Développement de méthodes rapides pour le calcul de structures électroniques, Ph.D. Thesis, Ecole Nationale des Ponts et Chaussées, 2005.

[2] M. Barrault, Y. Maday, N. C. Nguyen, A.T. Patera, An "empirical interpolation" method: Application to efficient reduced-basis discretization of partial differential equations, C. R. Acad. Sci. Paris, Serie I., 339 (2004) 667-672.

[3] E. Cancès, C. Le Bris, Y. Maday, G. Turinici, Towards reduced basis approaches in ab initio electronic structure computations, J. Sci. Comput., 17 (2002), no. 1-4, 461-469

[4] E. Cancès, M. Defranceschi, W. Kutzelnigg, C. Le Bris, Y. Maday, Computational quantum chemistry : a primer, in : Handbook of numerical analysis. Volume X : special volume : computational chemistry, Ph. Ciarlet and C. Le Bris (eds), North Holland 2003.

[5] E. Cancès, C. Le Bris, Y. Maday, N. C. Nguyen, A. T. Patera, G. S. H. Pau, Feasibility and competitiveness of a reduced-basis approach for rapid electronic structure calculations in quantum chemistry, In proceeding of the Workshop for High-dimensional Partial Differential in Science and Engineering (Montreal), 2007. To appear.

[6] L. Machiels, Y. Maday, I. B. Oliviera, A.T. Patera, D. V. Rovas, Outputs bounds for the reduced-basis approximations of symmetric positive definite eigenvalue problems, C. R. Acad. Sci. Paris, Serie I., 331 (2) (2000) 153-158.

[7] A. T. Patera, G. Rozza, Reduced basis approximation and a posteriori error estimation for parametrized partial differential equations, to appear in MIT Pappalardo Graduate Monographs in Mechanical Engineering.

[8] G. S. H. Pau, Reduced-basis method for quantum models of periodic solids, Ph.D. thesis, Massachusetts Institute of Technology, 2007.

[9] A. Pinkus, n-Widths in Approximation Theory, Springer-Verlag, 1985.

[10] C. Prud'homme, D. Rovas, K. Veroy, Y. Maday, A.T. Patera, G. Turinici, Reliable real-time solution of parametrized partial differential equations: Reduced basis output bound methods, J. Fluids Engrg. 124 (1) (2002) 70-80.

[11] E. M. Rønquist, A. E. Løvgren, Y. Maday, A reduced basis element method for the steady Stokes problem, M2AN Math. Model. Numer. Anal. 40 (3) (2006) 529-552.h

[12] K. Veroy, A.T. Patera, Certified real-time solution of the parametrized steady incompressible Navier-Stokes equations: rigourous reduced basis a posteriori error-bounds, Internat. J. Numer. Methods Fluids 47 (8-9) (2005) 773-788. 